Efeito Feedback Trading em Criptomoedas com Dados de Alta Frequência

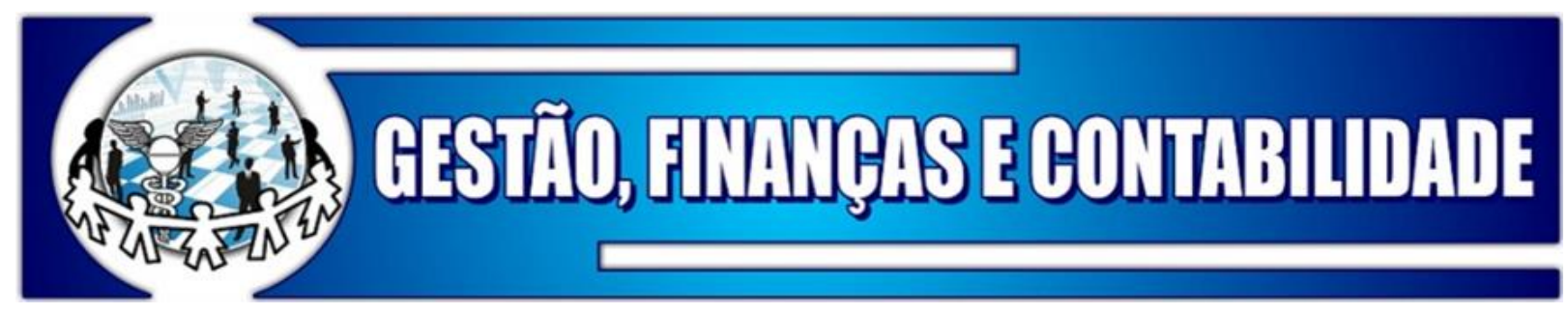

\title{
EFEITO FEEDBACK TRADING EM CRIPTOMOEDAS COM DADOS DE ALTA FREQUÊNCIA
}

\section{FEEDBACK TRADING EFFECT IN CRYPTOCURRENCIES WITH HIGH FREQUENCY DATA \\ EFECTO FEEDBACK TRADING SOBRE CRIPTOMOEDAS CON DATOS DE ALTA FRECUENCIA}

\author{
Cláudia Cristina Bozza \\ http://orcid.org/0000-0002-3954-0579 \\ MBA em Management (PUC-Rio) \\ Mestre em Administração, Concentração em Finanças (PUC-Rio) \\ E-mail: claudia.bozza@gmail.com
}

Marcelo Cabús Klotzle

http://orcid.org/0000-0002-5463-6333

Professor da Pontifícia Universidade Católica do Rio de Janeiro Coordenador Núcleo de Pesquisa em Finanças do IAG (PUC-Rio) Doutor em Economia (Katholische Universität Eichstätt - Alemanha) Pós-doutorado em Finanças Comportamentais (McMaster University - Canadá)

E-mail: klotzle@iag.puc-rio.br

Antônio Carlos Figueiredo Pinto http://orcid.org/0000-0002-1452-1240

Professor do Instituto de Administração e Gerência (IAG/PUC-Rio)

Coordenador do MBA em Finanças Corporativas (PUC-Rio) Doutor em Economia pela EPGE da Fundação Getúlio Vargas - RJ

E-mail: figueiredo@iag.puc-rio.br

Paulo Vitor Jordão da Gama Silva http://orcid.org/0000-0003-0598-4419

Professor da UNIGRANRIO

Membro do Núcleo de Pesquisa em Finanças (FINE/PUC-Rio) Membro da Associação de Peritos Judiciais do Estado do Rio de Janeiro (APJERJ) Doutor em Administração de Empresas com ênfase em Finanças (PUC-Rio) E-mail: paulo.jordao@ unigranrio.edu.br

\section{RESUMO}

Este trabalho buscou avaliar a existência do efeito de feedback trading para as criptomoedas Bitcoin, Ethereum, Litecoin e Dash usando o modelo VAR proposto por Hasbrouck (1991). 
Este efeito busca avaliar a utilização de dados passados para tomar decisões futuras, utilizando para tanto, dados de alta frequência, divididos em quatro períodos (dia, hora, minuto e segundo) para captar a existência do efeito de feedback trading nas criptomoedas, visando contribuir para a linha de finanças comportamentais, uma vez que há poucos estudos que avaliam o investimento em mercados digitais seguindo uma perspectiva comportamental. $\mathrm{O}$ resultado do modelo indica a existência de feedback trading negativo para todas as criptomoedas nas granularidades de tempo segundo e minuto. O estudo também aponta como resultado do modelo a existência de feedback trading negativo para a granularidade de tempo hora a hora para Litecoin e Dash.

Palavras-chave: Criptomoedas; Feedback Trading; Modelo VAR.

\section{ABSTRACT}

This study aimed to evaluate the feedback trading effect in cryptoassets Bitcoin, Ethereum, Litecoin and Dash, using a vector autoregressive system as proposed by Hasbrouck (1991). This effect seeks to evaluate the use of past data to make future decisions, using high frequency data, divided into four periods (day, hour, minute and second) in order to analyse the Feedback trading in cryptocurrencies and it intends to collaborate for the subfield of Behavioral finance, once there are few studies evaluating the investment in digital market under a behavioral perspective. The result suggest that there is a negative feedback trading effect in all cryptocurrencies for models using data aggregated by second and minute. The results also indicates that for Litecoin and Dash there is a negative feedback trading effect for data aggregated by hour.

Keywords: Cryptocurrencies; Feedback Trading; VAR Model.

\section{RESUMEN}

Este trabajo buscó evaluar la existencia del efecto de feedback trading para las criptomoedas Bitcoin, Ethereum, Litecoin y Dash usando el modelo VAR propuesto por Hasbrouck (1991). Este efecto busca evaluar la utilización de datos pasados para tomar decisiones futuras, utilizando para ello datos de alta frecuencia, divididos en cuatro períodos (día, hora, minuto y segundo) para captar la existencia del efecto de feedback trading en las criptomedas, con el fin de contribuir a para la línea de las finanzas del comportamiento, ya que hay pocos estudios que evalúan la inversión en mercados digitales siguiendo una perspectiva de comportamiento. El resultado del modelo indica la existencia de feedback trading negativo para todas las criptomoedas en las granularidades de tiempo segundo y minuto. El estudio también apunta como resultado del modelo la existencia de feedback trading negativo para la granularidad de tiempo hora a hora para Litecoin y Dash.

Palabras clave: Criptomoedas; Feedback Trading; Modelo VAR.

\section{INTRODUÇÃO}

Apesar do crescente interesse de investidores por criptomoedas, há poucos estudos acadêmicos que avaliam o investimento em mercados digitais seguindo uma perspectiva comportamental. Este trabalho busca, portanto, contribuir para a linha de finanças comportamentais avaliando a existência do efeito de feedback trading para criptomoedas usando o modelo VAR proposto por Hasbrouck (1991), uma avaliação inédita até o momento.

O feedback trading está relacionado a estratégias de negociação baseadas na busca de padrões de preço no passado. Esta estratégia pode estar relacionada a motivos comportamentais como, por exemplo, viés de disponibilidade (quando indivíduos julgam a 
Efeito Feedback Trading em Criptomoedas com Dados de Alta Frequência

frequência ou a probabilidade de um evento pela facilidade com que lembram de exemplos) e heurística da representatividade (evidenciando uma tendência em realizar julgamentos por meio de estereótipos) - um exemplo desta heurística é acreditar que o bom desempenho de uma empresa no passado é representativo de um desempenho geral que a empresa terá no futuro.

O feedback trading positivo, que chama maior atenção dos principais estudos, pode ser entendido como o comportamento errático de mercado, em que o investidor compra (vende) na baixa e vende (compra) na alta. Como visto em Silva (2020), neste fenômeno, além de investigar se o investidor utiliza informações passadas para tomar decisões futuras (similar ao fenômeno de momentum), o foco também está na tomada decisões dos investidores (que podem atuar de maneira mais racional ou errática).

As criptomoedas utilizam criptografia em seus algoritmos, o que viabiliza a criação de novos ativos, bem como a validação de transações feitas para compra e venda. Entre as vantagens desses novos ativos estão o fato de ser um modelo descentralizado, com menos riscos de fraude e pelas transações ocorrerem de forma mundial, sem barreiras entre países.

Por esses pontos positivos e pela crescente valorização desses ativos, tem aumentado o interesse de investidores em realizar transações no mercado digital e a hipótese deste estudo é de que em investimentos em ativos mais populares, como o Bitcoin, seja possível identificar um efeito maior de feedback trading.

Para esta análise, será utilizado o modelo VAR proposto por Hasbrouck (1991), apontado em outros estudos acadêmicos como uma solução para avaliar o efeito de feedback trading. Este estudo usa dados de alta frequência, divididos em quatro períodos (dia, hora, minuto e segundo) para captar esse efeito. Este trabalho busca entender como o feedback trading se manifesta nas criptomoedas Bitcoin, Ethereum, Litecoin e Dash, pela relevância desses ativos no mercado digital em volume e em capitalização de mercado.

A estrutura deste trabalho divide-se em quatro seções. A primeira apresenta o referencial teórico, abordando estudos sobre feedback trading e o modelo VAR proposto por Hasbrouck (1991) e a aplicação do modelo VAR para avaliação de feedback trading. A segunda apresenta a metodologia usando VAR para análise de feedback trading em criptomoedas. A terceira apresenta as análises e resultados encontrados e, por fim, na quarta apresentam-se as conclusões do estudo.

\section{REFERENCIAL TEÓRICO}

No campo financeiro, teóricos das finanças comportamentais com bases na psicologia começam a ganhar espaço com alguns estudos como os de Simon (1955), Muth (1961), Weintraub (1963), Lease, Lewellen e Schlarbaum (1974) e Kahneman e Tversky (1979), tendo estes últimos autores focado na teoria do prospecto em resposta a teorias normativas sobre o processo de tomada de decisão em contextos econômico-financeiros, bem como, em Kahneman e Tversky (1986), no qual os mesmos autores comentam sobre escolhas racionais e tomada de decisão, no qual argumentam que os desvios do comportamento real dos indivíduos em relação aos modelos normativos são muito generalizados para serem ignorados, bem como muito sistemáticos para serem desconsiderados como erros aleatórios.

Nesse contexto, estudos na área de finanças comportamentais ganharam espaço principalmente no meio internacional, com uma literatura vasta a respeito de tomada de risco, momentum, análise de excesso de confiança (overconfidence), efeitos emocionais e até mesmo a ideia de neurofinanças (abordando questões cognitivas do comportamento do indivíduo). No Brasil, o campo de finanças comportamentais vem crescendo, como visto em Silva, Santos e Pereira (2019), com o intuito de entender o que direciona o comportamento dos investidores 
nos mercados emergentes, de maneira a auxiliar estudos macroeconômicos, agências de ratings, companhias de corretagem, bancos e demais empresas.

Como observado em Gallo (2016), tais relações comportamentais que violam pressupostos da eficiência do mercado contribuem para um aumento da complexidade e subjetividade nesta dinâmica. É importante acrescentar que mesmo com um aspecto regulatório consolidado, modelos de precificação de ativos bem definidos, mapeamento de vieses relacionais entre economias e entre os principais players (de comportamento de contágio, manada, entre outros), é possível observar a influência dos fenômenos comportamentais em produtos que pertencem a um mercado consolidado. Como será esse efeito em um mercado com produtos altamente voláteis e recentes tal qual o de criptomoedas? É esperado que tais fenômenos sejam muito mais proeminentes? Como isso pode afetar os investidores neste mercado?

\subsection{Formação de Preço de Criptomoedas}

A alta volatilidade de preços do Bitcoin desde seu surgimento no mercado em 2009 até os dias atuais não é um comportamento comum entre moedas convencionais. Essa volatilidade atípica sugere a existência de fatores específicos na formação de preço de moedas e ativos digitais.

No intuito de entender tais fatores, estudos foram feitos para avaliar o impacto de diferentes determinantes na formação de preço do Bitcoin, como fatores tradicionais de oferta e demanda que afetam o movimento de preços de moedas convencionais, fatores específicos para ativos digitais, como a atratividade da criptomoeda para investidores, e por fatores macrofinanceiros.

Buchholz et al. (2012), por exemplo, investigam o impacto da oferta e demanda no movimento de preços do Bitcoin. Os autores apontaram que, assim como para moedas convencionais, grande parte do movimento de preços do Bitcoin pode ser explicado pela interação entre oferta e demanda da criptomoeda.

Por outro lado, Van Wijk (2013) estuda o movimento de preços do Bitcoin com uma perspectiva macrofinanceira. Segundo o autor, no longo prazo, fatores como o índice Dow Jones, a taxa de câmbio Euro x Dólar e o preço do petróleo têm uma relação significativa em relação ao valor do Bitcoin.

Para Bouoiyour et al. (2016) o movimento de preços do Bitcoin é altamente desassociado de fatores macroeconômicos e apresenta um comportamento mais semelhante ao de uma bolha especulativa. Evidências encontradas pelos autores mostram que a especulação (fator usado como uma proxie para a atratividade dos investidores pelo Bitcoin) é um forte determinante de preço para o Bitcoin, superando o impacto de fatores como as forças de oferta e demanda do mercado.

Pavel, Miroslava e D'Artis (2015) investigaram o movimento de preços do Bitcoin avaliando tanto determinantes tradicionais de formação de preço de moedas convencionais, quanto determinantes específicos para o mercado digital. $\mathrm{O}$ estudo dos autores evidenciou os seguintes aspectos para a formação de preço desta criptomoeda:

- Como encontrado por Buchholz et al. (2012), oferta e demanda por Bitcoin tem um importante papel no preço do criptoativo. A conclusão dos autores aponta que grande parte da formação de preço do Bitcoin pode ser explicada por um modelo econômico padrão de formação de preço de moedas;

- Os autores apontaram também que novas informações afetam positivamente o preço do Bitcoin. Além disso, eles concluem que, inicialmente, quando o Bitcoin era pouco conhecido entre os investidores, as buscas por informação sobre Bitcoin (visualizações 
na Wikipedia foram usados como proxie para esse fator) exerciam forte impacto no preço do Bitcoin. No longo prazo, entretanto, as visualizações na Wikipedia não impactaram mais no preço da criptomoeda;

- Os autores os autores não encontraram indícios suficientes que permitissem rejeitar a hipótese de que especulações afetam o preço do Bitcoin. Segundo Pavel, Miroslava e D'Artis (2015), o impacto de visualizações na Wikipedia e conteúdos sobre Bitcoin são estatisticamente significativos a curto prazo e pode indicar um comportamento especulativo dos investidores;

- Por fim, ao contrário das descobertas apontadas por Van Wijk (2013), os autores não encontraram evidências que apontem relevante impacto dos determinantes macrofinanceiros no preço do Bitcoin no longo prazo. Segundo os autores, o índice Dow Jones, a taxa de câmbio Euro x Dólar e o preço do petróleo são significativos apenas no curto prazo.

\subsection{Feedback Trading: Principais Modelos e Estudos na Área}

O impacto de feedback traders no equilíbrio dos preços tem sido tema de estudos em finanças ao longo dos anos, principalmente com a perspectiva de estratégias de feedback trading positivo, em que os investidores compram (vendem) quando preços sobem e vendem (compram) quando os preços caem, baseados em tendências de mercado (utilizando dados passados para análise).

Muitos modelos sobre feedback trading foram propostos para avaliar padrões de autocorrelação dos retornos, sendo os modelos de Hasbrouck (1991) e de Sentana e Wadhwani (1992) os principais desenvolvidos e de ampla utilização - até mesmo pelo caráter seminal. Porém, enquanto o primeiro autor trabalha com dados de alta frequência, os dois autores do modelo posterior trabalham com dados diários.

Cohen e Shin (2003) analisam a existência de feedback trading positivo no mercado de títulos do Tesouro Americano, utilizando alterações de cotação e negociações assinadas de notas do Tesouro dos EUA de 2 anos, 5 anos e 10 anos em circulação. A investigação utiliza o modelo VAR proposto por Hasbrouck (1991) com o intuito de saber se a instituição pode ser substanciada a partir de dados de mercado. Os autores concluem que em condições tranquilas de mercado, com negociações ordenadas e com baixa frequência de negociação, é possível replicar grande parte das conclusões qualitativas encontradas para o mercado de ações. Segundo os autores, a principal diferença encontrada por eles e por Hasbrouck é que Hasbrouck conclui que as mudanças de preços passadas geralmente têm um efeito negativo no fluxo de compra.

No trabalho de Cohen e Shin isso só foi evidenciado para notas de 10 anos. Para as notas de dois e cinco anos, o efeito é significativo e positivo. Outra conclusão de Cohen e Shin (2003) é que em períodos de alta volatilidade de preços e de transações ativas, parece haver uma mudança na dinâmica do mercado. Segundo os autores, nesses períodos é reforçado o efeito positivo do fluxo de compra passado no preço atual e vice-versa. Assim, ordens de compra provocam preços mais altos e o aumento de preços provoca mais ordens de compra, tornando o movimento dos preços mais positivamente autocorrelacionados em horizontes curtos. Isso ocorre, apesar das negociações assinadas tenderem a ser menos positivamente autocorrelacionadas nesses períodos.

Danielsson e Love (2006) utilizaram o modelo de Hasbrouck (1991) para analisar o spot USD/EUR no mercado de câmbio internacional com o intuito de avaliar a importância do parâmetro de feedback trading na estimativa de preços em fluxos transacionais. Os autores consideraram duas amostras com frequências de um e cinco minutos. A partir dessas amostras eles estimaram VARs com e sem feedback trading. Os autores encontraram dependência 
positiva dos retornos com os fluxos de transações contemporâneos. Eles concluem também que o fluxo de transações parece depender positivamente dos retornos contemporâneos, sugerindo que os agentes compram a moeda no mesmo período, possivelmente porque eles esperam que o preço varie na mesma direção. Segundo os autores, os resultados do VAR para frequências maiores que um minuto são similares, mas mais acentuados.

Os autores Chang e Ke (2014) também utilizam o modelo VAR para investigar a relação entre fluxos e retornos de cinco fundos de investimentos ETF (do inglês Exchange Traded Funds) do setor de energia dos Estados Unidos. Os autores analisam quatro hipóteses sobre o setor, incluindo hipótese sobre pressão do preço, hipótese de informação, hipótese de feedback trading e hipótese de suavização. A conclusão é que os retornos de energia e os fluxos subsequentes de ETF de energia apresentam uma relação negativa, suportando a hipótese de suavização. Os autores apontam também que o efeito de suavização ficou evidenciado para XLE e IYE durante a crise financeira global. Contudo, não foram encontradas evidências para as hipóteses de pressão de preço, hipótese de informação ou hipótese de feedback trading.

Chau, Kuo e Shi (2015), por exemplo, estudaram a presença de feedback trading no mercado de emissões e energia e até que ponto esse comportamento está associado a uma oportunidade de arbitragem utilizando o modelo proposto por Sentana e Wadhwani (1992). Os autores encontraram evidências de feedback trading nos mercados de carvão e eletricidade na Europa, mas não identificaram tal comportamento no mercado de carbono, onde instituições de investimento dominam as transações. Para os autores, a conclusão do estudo é consistente com a premissa de que instituições de investimento são menos suscetíveis a adotar estratégias de feedback trading. Os autores também concluíram que o efeito de feedback trading está relacionado ao nível de oportunidades de arbitragem e que a intensidade dessa relação está associada ao tipo de regime do mercado.

Também utilizando o modelo proposto por Sentana e Wadhwani (1992), Bohl, Klein e Siklos (2014) estudaram a relação entre vendedores a descoberto (do inglês short sellers) e feedback traders positivos. Durante a crise financeira global, entre 2008-2009, políticos, reguladores e a mídia culparam a atuação de short sellers na ampliação da desaceleração do mercado de ações. Nesse cenário, os autores investigaram os regimes de venda a descoberto no Reino Unido, EUA, Alemanha, França, Coréia do Sul e Austrália durante o período da crise financeira global e concluíram que para a maioria dos mercados estudados as restrições à venda a descoberto aumentam o efeito de feedback trading positivo durante um período de alta volatilidade, ao invés de mitigá-lo. Com isso, os autores apontam que as proibições de vendas a descoberto não contribuem para aumentar a estabilidade financeira.

Chau, Deesomsak e Lau (2011) ampliaram o modelo de feedback trading proposto por Sentana e Wadhwani (1992) com o intuito de incluir a variável de sentimento na demanda por ações por feedback traders. O objetivo dos autores era investigar em qual grau o sentimento dos investidores influencia no nível de feedback trading em três grandes ETFs nos Estados Unidos. Os autores concluíram que existe o efeito de feedback trading positivo nesses mercados e que a intensidade, geralmente, está ligada ao sentimento do investidor, principalmente quando os investidores estão otimistas.

Bohl e Reitz (2004) investigaram a influência de feedback trading positivo no retorno do mercado de ações da Alemanha a partir do modelo proposto por Sentana e Wadhwani (1992). Os autores concluíram que uma parcela significativa dos investidores são feedback traders positivos e que o efeito do feedback trading positivo aparenta ter uma autocorrelação negativa com o retorno durante períodos de alta volatilidade no mercado alemão.

\subsection{O Modelo VAR Proposto por Hasbrouck}


Segundo Hasbrouck (1991), a percepção de que em um mercado em que haja agentes com assimetria de informação e que as negociações transmitem informação e, consequentemente, provocam um impacto no preço do ativo é crucial para análises de microestrutura de mercado. Para o autor, a magnitude do efeito do preço geralmente é uma função positiva sobre a proporção de potenciais negociadores informados, a probabilidade de que esses negociadores tenham de fato informação e a precisão da informação privada. Nesse cenário, o autor teve como objetivo determinar o impacto da assimetria de informação, estabelecendo uma caracterização da dinâmica pela qual as cotações e negociações interagem.

Para Hasbrouck (1991), as teorias sobre a extensão da assimetria de informação geram duas previsões empíricas: a primeira, que a assimetria é positivamente relacionada ao ganho (ou spread) e a segunda, que a assimetria é positivamente relacionada ao impacto do preço de uma transação. $\mathrm{O}$ autor sugere que as interações entre negociações de títulos e revisões de cotações podem ser modeladas por um sistema de vetor autoregressivo que permite avaliar a relação entre os retornos dos ativos e atividades de negociação, representado nas equações $1 \mathrm{e}$ 2.

$$
\begin{gathered}
R_{t}=\alpha_{1}+\beta F_{t}+\varphi_{11} R_{t-1}+\varphi_{12} F_{t-1}+\varepsilon_{t}^{R} \\
F_{t}=\alpha_{2}+\varphi_{21} R_{t-1}+\varphi_{22} F_{t-1}+\varepsilon_{t}^{F}
\end{gathered}
$$

Onde:

- $R_{t}$ é o retorno do ativo no período t, definido como o log da primeira diferença de

preços;

- $F_{t}$ é o fluxo de transação no período t, definido como o número de compradores menos

vendedores que iniciaram transações no intervalo de tempo;

- $\varepsilon_{t}^{R}$ e $\varepsilon_{t}^{F}$, erros independentes, não correlacionados em série, com variância $\theta_{g}^{2}$ e $\theta_{g}^{2}$,

respectivamente.

Com esse modelo, o autor chega às seguintes conclusões:

- O impacto total de uma transação no preço do ativo não é sentido instantaneamente, mas com um atraso prolongado;

- Como uma função do tamanho da inovação na transação, o impacto final da inovação na cotação é não-linear, positivo e crescente, porém, côncavo; O fluxo de compra é afetado por revisões anteriores da cotação;

- O tamanho do ganho demonstra uma resposta à atividade de transação. Transações maiores são particularmente associadas com um maior ganho;

- Transações que ocorrem frente a ganhos relativamente amplos apresentam maior impacto no preço do que aquelas que ocorrem quando os ganhos são menores;

- Entre empresas, o impacto do preço e a extensão da assimetria da informação é mais significativo para empresas com menor valor de mercado.

\section{METODOLOGIA}

\subsection{O Modelo VAR para Criptomoedas}

Este trabalho, portanto, utilizou o modelo VAR proposto por Hasbrouck (1991) para analisar o efeito de feedback trading para o Bitcoin, Ethereum, Litecoin e Dash e comparar a diferença do efeito entre eles. Estas criptomoedas foram selecionadas para este estudo pela 
relevância delas tanto pelo maior volume negociado, quanto capitalização de mercado existente atualmente e pela diversidade entre elas - em termos estratégicos, e de tempo no mercado. Tais diferenças podem atrair perfis distintos de investidores e, consequentemente, serem impactados de forma diversa pelo efeito de feedback trading.

O modelo estatístico de Hasbrouck (1991), como demonstrado anteriormente, permite avaliar a relação entre os retornos dos ativos e atividades de negociação, como apontado anteriormente nas equações 1 e 2 .

Foram utilizados os preços das criptomoedas para o cálculo do retorno, onde retorno $=\ln \left(\right.$ preço $_{t} /$ preço $\left._{t-1}\right)$ e os volumes de compras e vendas para calcular o volume de compradores menos vendedores, onde Volume $=$ Compras - Vendas.

O modelo requer dados de alta frequência e, portanto, as transações foram agregadas em hora, dia, minuto e segundo. Foram separados quatro grupos com diferentes frequências para cada criptomoeda, com o intuito de avaliar se a granularidade da frequência dos dados potencializa o efeito de feedback trading.

Essa diferença de impacto, dependendo da granularidade da base de dados, foi encontrada no estudo de Danielsson e Love (2006), citado anteriormente. Segundo os autores, os resultados do VAR para frequências menos granulares que um minuto são similares, porém são mais evidenciadas.

Este trabalho avalia o efeito de feedback trading utilizando uma variável dummy, como proposto por Hasbrouck (1991), que indica a direção da transação, sendo +1 , para uma compra e -1, para uma venda. Como no trabalho de Hasbrouck (1991), caso o volume seja < 0 , então -1 , volume $>0,+1$, volume $=0$, então 0 . Neste trabalho, utiliza-se VAR com lag 1 , visto que para dados de alta frequência o lag 1 é satisfatório para analisar o efeito de feedback trading nas criptomoedas.

Os dados deste trabalho foram extraídos da corretora Kraken via API (Application Programming Interface) disponibilizada pelo marketplace. A Kraken é sediada nos Estados Unidos e opera no Canadá, União Europeia, Japão e EUA, sendo bastante relevante em termos de volume e liquidez para Euro, além de ser a empresa que fornece informação de preço para a Bloomberg.

A Kraken fornece uma REST API onde após "pair=" deve ser inserida a sigla do criptoativo e após "since=" deve ser inserido o timestamp inicial do período que se deseja extrair os dados. O resultado dessa API é um json (um acrônimo de JavaScript Object Notation) que contém informações sobre as transações, temporalmente ordenadas, e um parâmetro chamado "last" que contém o timestamp da última transação.

Para extrair a próxima sequência de transações, o "since=" deve conter o "last" da última requisição. Cada requisição a essa API retorna um número limitado de transação, sendo necessário gerar uma função que permita extrair essas informações de forma mais automática, viabilizando processar e armazenar dados para um intervalo extenso de tempo. No apêndice 1 deste trabalho encontra-se um link para um arquivo armazenado em um Google Drive que contém o código em Python utilizado neste trabalho para armazenar os dados de criptomoedas.

Assim, para cada transação a corretora disponibiliza o timestamp com as seguintes informações: se foi uma transação de compra ou venda; o preço da criptomoeda; o volume comprado ou vendido na transação; e se a transação foi limit (por um preço fixo ou melhor) ou market (melhor média de preço de mercado). Para este trabalho, foram utilizadas apenas transações limit, pois além dessa opção contar com o maior número de transações para todas as criptomoedas, ainda há a hipótese de que este dado é mais qualificado para avaliar a existência de feedback trading, uma vez que expressa a aposta do investidor sobre a 
Efeito Feedback Trading em Criptomoedas com Dados de Alta Frequência

criptomoeda. Outro filtro para este trabalho foi a escolha do dólar como moeda de compra e venda das criptomoedas.

Este trabalho utilizou dados dos seguintes períodos para gerar o modelo: Bitcoin (201310-07 a 2018-09-20); Ethereum (2015-08-07 a 2018-09-20); Litecoin (2013-11-04 a 2018-0920) e Dash (2017-04-12 a 2018-09-21). Para as agregações de segundo, minuto, hora e dia, foram somados os volumes das criptomoedas compradas e vendidas e o preço escolhido para a agregação foi o preço do maior timestamp daquele intervalo de tempo.

\section{RESULTADOS}

\subsection{Bitcoin}

Como aponta o sumário estatístico presente na Tabela 1, a média do retorno logarítmico é próxima a zero e a volatilidade do retorno logarítmico do ativo é alta, para as agregações de tempo segundo (3,776,169 observações), minuto (862,130 observações), hora $(31,623$ observações) e dia (1,794 observações). A média de preço do ativo ficou em $\$ 6,287.95$ (agregação segundo), \$5,259 (agregação minuto), \$3,015.22 (agregação hora) e \$2,339.10 (agregação dia), com alta volatilidade de preços para todas as agregações de tempo - apenas recordando que a variação em função da agregação de tempo busca entender melhor se a granularidade de frequência pode potencializar o efeito comportamental que está se avaliando.

Tabela 1 - Sumário Estatístico do Bitcoin

\begin{tabular}{c|c|c|c|c|c|c|c|c}
\hline & \multicolumn{2}{|c|}{ Segundo } & \multicolumn{2}{c|}{ Minuto } & \multicolumn{2}{c|}{ Hora } & \multicolumn{2}{c}{ Dia } \\
\hline & Retorno & Preço & Retorno & Preço & Retorno & Preço & Retorno & Preço \\
\hline count & \multicolumn{2}{|c|}{$3,776,169$} & \multicolumn{2}{|c|}{862,130} & \multicolumn{2}{c}{31,623} & \multicolumn{2}{c}{1,794} \\
\hline mean & $\$ 0$ & $\$ 6,287.95$ & $\$ 0.00001$ & $\$ 5,259$ & $\$ 0.00015$ & $\$ 3,015.22$ & $\$ 0.00034$ & $\$ 2,339.10$ \\
\hline std & $\$ 0.003$ & $\$ 4,286.25$ & $\$ 0.00569$ & $\$ 4,212$ & $\$ 0.03004$ & $\$ 3,792.65$ & $\$ 0.10798$ & $\$ 3,484.26$ \\
\hline min & $-\$ 3.962$ & $\$ 123.61$ & $-\$ 3.94545$ & $\$ 124$ & $-\$ 3.94545$ & $\$ 123.61$ & $-\$ 3.94545$ & $\$ 123.61$ \\
\hline $\mathbf{2 5 \%}$ & $\$ 0$ & $\$ 2,479.84$ & $-\$ 0.00049$ & $\$ 1,204$ & $-\$ 0.00347$ & $\$ 454.70$ & $-\$ 0.01494$ & $\$ 379.19$ \\
\hline $\mathbf{5 0 \%}$ & $\$ 0$ & $\$ 6,452.50$ & $\$ 0.00000$ & $\$ 4,406$ & $\$ 0.00000$ & $\$ 826.70$ & $\$ 0.00229$ & $\$ 620.90$ \\
\hline $\mathbf{7 5 \%}$ & $\$ 0$ & $\$ 9,140.00$ & $\$ 0.00053$ & $\$ 8,026$ & $\$ 0.00395$ & $\$ 5,784.65$ & $\$ 0.01983$ & $\$ 2,547.26$ \\
\hline $\mathbf{m a x}$ & $\$ 0.304$ & $\$ 19,660.00$ & $\$ 0.31649$ & $\$ 19,660$ & $\$ 2.39641$ & $\$ 19,539.30$ & $\$ 1.11791$ & $\$ 19,356.90$ \\
\hline
\end{tabular}

Fonte: Elaborada pelos autores.

Os dados apresentados na Tabela 2 apontam que para o Bitcoin os resultados são diferentes se a base de dados for agregada por segundo, minuto, hora ou dia.

O modelo gerado pela agregação de tempo segundo a segundo evidência que existe uma autocorrelação positiva significativa nas transações, evidenciado pelo lag do coeficiente das transações na estimativa da equação de transações. Existe também uma autocorrelação negativa significativa entre retornos, evidenciado pelo lag do coeficiente do retorno na estimativa da equação de retorno.

Tabela 2 - Resultado do Modelo VAR proposto por Hasbrouck (1991) para o Bitcoin

\begin{tabular}{l|c|c|c|c|c|c|c|c|c}
\hline & \multicolumn{2}{c}{ Segundo } & \multicolumn{2}{c}{ Minuto } & \multicolumn{2}{c}{ Hora } & \multicolumn{2}{c}{ Dia } \\
\hline & Coeficiente & Prob & Coeficiente & Prob & Coeficiente & Prob & Coeficiente & Prob \\
\hline Resultado da Equação de Transações (dummy) \\
\hline Trans & $0.922691 * * *$ & 0.000 & $0.460673 * * *$ & 0.000 & $0.216461 * * *$ & 0.000 & $0.171288 * * *$ & 0.000 \\
\hline$\underline{\text { Retorno }}$ & $\underline{2.747 \overline{7} 99 * * *}$ & $\underline{0.000}$ & $\underline{3.558732 * * *}$ & $\underline{0.000}$ & $\underline{-0.337471}$ & $\underline{0.100}$ & $\underline{-0.285207}$ & $\underline{0.186}$ \\
\hline
\end{tabular}


Efeito Feedback Trading em Criptomoedas com Dados de Alta Frequência

\section{Resultado da Equação de Retorno}

\begin{tabular}{l|c|c|c|c|c|c|c|c}
\hline Trans & -0.000002 & 0.107 & $0.000031 * * *$ & 0.000 & -0.000102 & 0.546 & 0.186 & 0.675 \\
\hline & - & & - & & - & & & \\
Retorno & $0.102846 * * *$ & 0.000 & $0.071323 * * *$ & 0.000 & $0.046377 * * *$ & 0.000 & -0.014498 & 0.542 \\
\hline
\end{tabular}

Código para nível de significância: $1 \%$ '***' $5 \%$ '**’ $10 \%$ '*'

Fonte: Elaborada pelos autores.

O resultado do modelo segundo a segundo está em linha com as descobertas empíricas feitas por Hasbrouck (1991). Como aponta o autor, as transações se mostraram positivamente autocorrelacionadas, sugerindo que compras tendem a levar a compras e vendas a vendas, e os retornos negativamente autocorrelacionados. Por outro lado, segundo Hasbrouck (1991), o lag de transações tende a exercer um efeito positivo no retorno em $\mathrm{t}$, ou seja, os movimentos de preços seguem o fluxo de compra, contudo esse efeito não foi encontrado no modelo segundo a segundo do Bitcoin.

O resultado do modelo também aponta para uma autocorrelação negativa significativa entre lag do retorno e transações em t, como aponta o coeficiente do lag do retorno na equação de transações, apontando a existência de feedback trading negativo para Bitcoin na agregação segundo a segundo.

Assim como o modelo segundo a segundo, o modelo gerado pela agregação minuto a minuto aponta que existe forte autocorrelação positiva nas transações, evidenciado pelo lag do coeficiente das transações na estimativa da equação de transações e uma autocorrelação negativa significativa entre retornos, evidenciado pelo lag do coeficiente do retorno na estimativa da equação de retorno.

Diferentemente do que ocorre com o modelo segundo a segundo, o resultado minuto a minuto aponta também uma significativa autocorrelação positiva nas transações e no retorno, como observa-se pelo coeficiente do lag das transações na estimativa da equação de retorno.

O resultado do modelo minuto a minuto está em linha com as descobertas feitas por Hasbrouck (1991). Para o autor, o lag de transações tende a exercer um efeito positivo no retorno em t, ou seja, os movimentos de preços seguem o fluxo de compra. Igualmente em linha com Hasbrouck (1991), as transações se mostraram positivamente autocorrelacionadas, sugerindo que compras tendem a levar a compras e vendas a vendas, e os retornos negativamente autocorrelacionados.

O resultado minuto a minuto também apresenta uma autocorrelação negativa significativa entre lag do retorno e transações em t, como aponta o coeficiente do lag do retorno na equação de transações, novamente evidenciando a existência de feedback trading negativo.

Nos resultados com granularidade menor de tempo (hora a hora e dia a dia), é mantido o padrão de autocorrelação positiva entre transações. Nos resultados hora a hora, observa-se uma autocorrelação negativa entre retornos, em linha com Hasbrouck (1991) e com os resultados encontrados nos dados minuto a minuto, mas o mesmo efeito não é encontrado na granularidade dia a dia. Não foi encontrado para as granularidades hora a hora e dia a dia o efeito negativo do retorno nas transações. Também não foi encontrada autocorrelação positiva de transações e retorno para hora a hora e dia a dia.

Por meio dos resultados, não foram observados o efeito de feedback trading positivo para Bitcoin para nenhuma granularidade de tempo e que existem diferenças na relação entre fluxo de transações e retorno dependendo de como os dados são agrupados. Os resultados segundo a segundo e minuto a minuto apontam que existe efeito de feedback trading negativo no Bitcoin, ou seja, ocorrem vendas seguidas de retornos positivos e compras após retornos negativos. 
Efeito Feedback Trading em Criptomoedas com Dados de Alta Frequência

\subsection{Ethereum}

Como aponta o sumário estatístico presente na Tabela 3, a média do retorno logarítmico é próxima a zero e a volatilidade do retorno logarítmico do ativo é alta, para as agregações de tempo segundo $(2,609,289$ observações), minuto (653,148 observações), hora $(23,408$ observações) e dia (11,132 observações). A média de preço do ativo ficou em $\$ 465.87$ (agregação segundo), \$390.46 (agregação minuto), \$245.24 (agregação hora) e \$213.28 (agregação dia), com alta volatilidade de preços para todas as agregações de tempo.

Tabela 3 - Sumário Estatístico do Ethereum

\begin{tabular}{c|c|c|c|c|c|c|c|c}
\hline & \multicolumn{2}{|c}{ Segundo } & \multicolumn{2}{c|}{ Minuto } & \multicolumn{2}{c|}{ Hora } & \multicolumn{2}{c}{ Dia } \\
\hline & Retorno & Preço & Retorno & Preço & Retorno & Preço & Retorno & Preço \\
\hline count & \multicolumn{2}{|c|}{$2,609,289$} & \multicolumn{2}{|c|}{653,148} & \multicolumn{2}{c}{23,408} & \multicolumn{2}{c}{1,132} \\
\hline mean & $\$ 0$ & $\$ 465.87$ & $\$ 0.00001$ & $\$ 390.46$ & $\$ 0.00022$ & $\$ 245.24$ & $-\$ 0.00015$ & $\$ 213.28$ \\
\hline Std & $\$ 0.006$ & $\$ 326.92$ & $\$ 0.00895$ & $\$ 297.53$ & $\$ 0.04578$ & $\$ 288.37$ & $\$ 0.17498$ & $\$ 282.72$ \\
\hline Min & $-\$ 4.321$ & $\$ 0.15$ & $-\$ 4.32059$ & $\$ 0.15$ & $-\$ 4.32059$ & $\$ 0.15$ & $-\$ 5.20410$ & $\$ 0.42$ \\
\hline $\mathbf{2 5 \%}$ & $\$ 0$ & $\$ 228.48$ & $-\$ 0.00079$ & $\$ 199.10$ & $-\$ 0.00568$ & $\$ 11.23$ & $-\$ 0.02951$ & $\$ 9.75$ \\
\hline $\mathbf{5 0 \%}$ & $\$ 0$ & $\$ 370.17$ & $\$ 0.00000$ & $\$ 315.48$ & $\$ 0.00000$ & $\$ 96.61$ & $\$ 0.00000$ & $\$ 18.69$ \\
\hline $\mathbf{7 5 \%}$ & $\$ 0$ & $\$ 691.97$ & $\$ 0.00082$ & $\$ 564.59$ & $\$ 0.00616$ & $\$ 403.16$ & $\$ 0.03616$ & $\$ 337.33$ \\
\hline $\mathbf{m a x}$ & $\$ 1.467$ & $\$ 1,451.00$ & $\$ 1.46692$ & $\$ 1,440.00$ & $\$ 2.59929$ & $\$ 1,435.00$ & $\$ 0.77122$ & $\$ 1,398.99$ \\
\hline
\end{tabular}

Fonte: Elaborada pelos autores.

Como ocorre com o Bitcoin, os resultados apresentados na Tabela 4 apontam que para o Ethereum há diferenças se a base de dados for agregada por segundo, minuto, hora ou dia.

$\mathrm{O}$ modelo gerado pela agregação segundo a segundo apontou as mesmas autocorrelações do resultado segundo a segundo do Bitcoin. Assim, ficou evidenciado que existe uma autocorrelação positiva significativa nas transações, evidenciado pelo lag do coeficiente das transações na estimativa da equação de transações e uma autocorrelação negativa significativa entre retornos, evidenciado pelo lag do coeficiente do retorno na estimativa da equação de retorno. O modelo apontou também a existência de autocorrelação negativa significativa entre lag do retorno e transações em t, como aponta o coeficiente do lag do retorno na equação de transações. Como ocorre com Bitcoin, não existe autocorrelação positiva para o modelo segundo a segundo entre o lag de transações na equação de retorno.

Tabela 4 - Resultado do Modelo VAR proposto por Hasbrouck (1991) para o Ethereum

\begin{tabular}{|c|c|c|c|c|c|c|c|c|}
\hline & \multicolumn{2}{|c|}{ Segundo } & \multicolumn{2}{|l|}{ Minuto } & \multicolumn{2}{|l|}{ Hora } & \multicolumn{2}{|l|}{ Dia } \\
\hline & Coeficiente & Prob & Coeficiente & Prob & Coeficiente & Prob & Coeficiente & Prob \\
\hline \multicolumn{9}{|c|}{ Resultado da Equação de Transações (dummy) } \\
\hline Trans. & $0.937097 * * *$ & 0.000 & $0.528712 * * *$ & 0.000 & $0.202034 * * *$ & 0.000 & $0.166062 * * *$ & 0.000 \\
\hline Retorno & $1.000 \overline{4} 81 * * *$ & $\underline{0.000}$ & $-2.033556 * * *$ & $\underline{0.000}$ & $\underline{-0.170531}$ & 0.229 & $\underline{-0.116053}$ & 0.497 \\
\hline \multicolumn{9}{|c|}{ Resultado da Equação de Retorno } \\
\hline Trans. & 0.00000 & 0.363 & $0.000105 * * *$ & 0.000 & 0.000020 & 0.946 & -0.004847 & 0.350 \\
\hline Retorno & $0.287735^{-} * * *$ & 0.000 & $-0.098972 * * *$ & 0.000 & 0.003047 & 0.646 & 0.041538 & 0.165 \\
\hline
\end{tabular}

Fonte: Elaborada pelos autores. 
Efeito Feedback Trading em Criptomoedas com Dados de Alta Frequência

O modelo gerado pela agregação minuto a minuto aponta que existe forte autocorrelação positiva nas transações, evidenciado pelo lag do coeficiente das transações na estimativa da equação de transações. $O$ resultado aponta também uma significativa autocorrelação positiva nas transações e no retorno, como observa-se pelo coeficiente do lag das transações na estimativa da equação de retorno e uma autocorrelação negativa significativa entre retornos, evidenciado pelo lag do coeficiente do retorno na estimativa da equação de retorno. O modelo também mostra que existe autocorrelação negativa significativa entre lag do retorno e transações em t, como aponta o coeficiente do lag do retorno na equação de transações. Os resultados do Ethereum para a agregação minuto a minuto, assim como ocorre com o Bitcoin, estão em linha com as descobertas de Hasbrouck (1991).

Os dados hora a hora do Ethereum diferem dos resultados encontrados hora a hora para Bitcoin e dos resultados minuto a minuto para o Ethereum. Os resultados hora a hora apontam que existe somente uma forte autocorrelação positiva nas transações, evidenciado pelo lag do coeficiente das transações na estimativa da equação de transações. Como visto anteriormente na análise de resultados do Bitcoin, observa-se que os retornos são negativamente autocorrelacionados, resultado condizente com Hasbrouck (1991). Contudo, esse efeito nos retornos não é significativo para o Ethereum para a granularidade de tempo hora a hora.

Os dados dia a dia apontam também que existe somente forte autocorrelação positiva nas transações, evidenciado pelo lag do coeficiente das transações na estimativa da equação de transações. Esse resultado para o modelo dia a dia também foi encontrado para o Bitcoin dia a dia e para os dados hora a hora do Ethereum.

Como encontrado na análise dos dados para Bitcoin, não foi observado o efeito de feedback trading positivo para Ethereum em nenhuma granularidade de tempo. O que se pode observar é a presença de feedback trading negativo apenas para os dados com granularidade segundo a segundo e minuto a minuto. Para outros agrupamentos de tempo, não existe efeito de feedback trading.

\subsection{Litecoin}

Como aponta o sumário estatístico presente na Tabela 5, a média do retorno logarítmico é próxima a zero e a volatilidade do retorno logarítmico do ativo é alta, para as agregações de tempo segundo (978,374 observações), minuto (356,697 observações), hora $(19,744$ observações) e dia (1,586 observações). A média de preço do ativo ficou em $\$ 106.85$ (agregação segundo), \$101.87 (agregação minuto), \$64.50 (agregação hora) e \$36.67 (agregação dia), com alta volatilidade de preços para todas as agregações de tempo.

Tabela 5 - Sumário estatístico do Litecoin

\begin{tabular}{|c|c|c|c|c|c|c|c|c|}
\hline & \multicolumn{2}{|c|}{ Segundo } & \multicolumn{2}{|c|}{ Minuto } & \multicolumn{2}{|c|}{ Hora } & \multicolumn{2}{|c|}{ Dia } \\
\hline & Retorno & Preço & Retorno & Preço & Retorno & Preço & etorno & Preço \\
\hline count & \multicolumn{2}{|c|}{978,374} & \multicolumn{2}{|c|}{356,697} & \multicolumn{2}{|c|}{19,744} & \multicolumn{2}{|c|}{1,586} \\
\hline mean & $\$ 0.000002$ & & $\$ 0.00001$ & & $\$ 0.00012$ & & $\$ 0.000$ & $\$ 36.67$ \\
\hline std & $\$ 0.007761$ & $\$ 77.68$ & $\$ 0.01224$ & $\$ \$ 77.20$ & $\$ 0.04777$ & $\$ 69.70$ & $\$ 0.13033$ & $\$ 58.58$ \\
\hline $\min$ & $-\$ 2.905078$ & $\$ 1.20$ & $\$ 2.90508$ & $\$ 1.20$ & $\$ 2.90508$ & $\$ 1.20$ & $\begin{array}{c}- \\
\$ 2.90508 \\
\end{array}$ & $\$ 1.20$ \\
\hline $25 \%$ & $-\$ 0.000049$ & $\$ 46.00$ & \begin{tabular}{|c|}
- \\
$\$ 0.00108$ \\
\end{tabular} & $\$ 45.68$ & $\begin{array}{c}- \\
\$ 0.00716 \\
\end{array}$ & $\$ 4.68$ & \begin{tabular}{|c|}
- \\
$\$ 0.03053$ \\
\end{tabular} & $\$ 3.69$ \\
\hline $50 \%$ & $\$ 0$ & $\$ 74.70$ & $\$ 0.00000$ & $\$ 68.70$ & $\$ 0.00000$ & $\$ 45.97$ & $\$ 0.00076$ & $\$ 5.10$ \\
\hline $75 \%$ & $\$ 0.000132$ & $\$ 163.00$ & $\$ 0.00111$ & $\$ 152.56$ & $\$ 0.00719$ & $\$ 87.70$ & $\$ 0.02724$ & $\$ 48.15$ \\
\hline
\end{tabular}


Efeito Feedback Trading em Criptomoedas

com Dados de Alta Frequência

\section{\begin{tabular}{l|l|l|l|l|l|l|l|l}
\hline $\max$ & $\$ 1.062445$ & $\$ 368.00$ & $\$ 1.06245$ & $\$ 367.50$ & $\$ 1.87643$ & $\$ 364.32$ & $\$ 1.16029$ & $\$ 353.65$ \\
\hline
\end{tabular}}

Fonte: Elaboração própria.

Os resultados encontrados pelo modelo segundo a segundo (Tabela 6) apresentam as mesmas autocorrelações dos resultados segundo a segundo para Bitcoin e Ethereum. $\mathrm{O}$ modelo segundo a segundo do Litecoin aponta que existe uma autocorrelação positiva significativa nas transações, evidenciado pelo lag do coeficiente das transações na estimativa da equação de transações e uma autocorrelação negativa significativa entre retornos, evidenciado pelo lag do coeficiente do retorno na estimativa da equação de retorno. O modelo apontou também a existência de autocorrelação negativa significativa entre lag do retorno e transações em t, como aponta o coeficiente do lag do retorno na equação de transações. Como ocorre com Bitcoin e Ethereum, não foi observada autocorrelação positiva para o modelo segundo a segundo entre o lag de transações na equação de retorno.

Tabela 6 - G Resultado do modelo VAR proposto por Hasbrouck (1991) para o Litecoin

\begin{tabular}{l|c|c|c|c|c|c|c|c}
\hline & \multicolumn{2}{|c|}{ Segundo } & \multicolumn{2}{c|}{ Minuto } & \multicolumn{2}{c|}{ Hora } & \multicolumn{2}{c}{ Dia } \\
\hline & Coeficiente & Prob & Coeficiente & Prob & Coeficiente & Prob & Coeficiente & Prob \\
\hline Resultado da Equação de Transações (dummy) \\
\hline Trans. & $0.958139^{* * *}$ & 0.000 & $0.641159^{* * *}$ & 0.000 & $0.273428^{* * *}$ & 0.000 & $0.157378^{* * * *}$ & 0.000 \\
\hline Retorno & $0.582366^{* * *}$ & 0.000 & $-1.586331 * * *$ & 0.000 & $0.452187 * * *$ & 0.002 & -0.170932 & 0.374 \\
\hline
\end{tabular}

Resultado da Equação de Retorno

\begin{tabular}{l|c|c|c|c|c|c|c|c}
\hline Trans. & 0.000007 & 0.336 & $0.000138 * * *$ & 0.000 & 0.000368 & 0.274 & -0.003104 & 0.338 \\
\hline Retorno & $0.166462 * * *$ & 0.000 & $-0.189757 * * *$ & 0.000 & $0.178968 * * *$ & 0.000 & $-0.167296^{* * * *}$ & 0.000 \\
\hline
\end{tabular}

Código para nível de significância: $1 \%$ '***’ $5 \%$ '**’ $10 \%$ ‘*’

Fonte: Elaboração própria.

Assim como ocorre no resultado encontrado para Bitcoin e Ethereum, o modelo minuto a minuto (Tabela 6) aponta que existe forte autocorrelação positiva entre volume de transações e o lag do volume de transações. Existe também forte autocorrelação positiva entre o lag do volume de transações e o retorno e forte autocorrelação negativa entre o lag do retorno e o retorno, em linha com as descobertas de Hasbrouck (1991).

Assim como ocorre com o Bitcoin e Ethereum, o modelo aponta também que existe forte autocorrelação negativa entre o lag do retorno e o fluxo de transação. Esse resultado aponta a existência de feedback trading negativo para Litecoin.

Os resultados para hora a hora (Tabela 6) diferem dos resultados encontrados para Bitcoin e Ethereum. O modelo aponta que existe forte autocorrelação positiva entre o lag do volume de transações e as transações; forte autocorrelação positiva entre o lag de transações e o retorno e forte autocorrelação negativa entre retornos, resultados em linha com o estudo de Hasbrouck (1991).

O modelo aponta ainda para a existência de forte autocorrelação negativa entre o lag do retorno e o fluxo de transação, evidenciando a existência de feedback trading negativo para Litecoin também para a agregação hora a hora.

Os resultados dos modelos dia a dia (Tabela 6) para Litecoin também diferem dos resultados encontrados nos modelos do Bitcoin e do Ethereum. Além da autocorrelação positiva entre transações, também presente nas criptomoedas analisadas anteriormente, existe forte autocorrelação negativa entre retornos.

Desta maneira, como encontrado na análise dos dados para Bitcoin e Ethereum, não foi encontrado o efeito de feedback trading positivo para Litecoin em nenhuma granularidade de 
Efeito Feedback Trading em Criptomoedas com Dados de Alta Frequência

tempo. Observa-se pelos dados a existência de feedback trading negativo nas granularidades segundo a segundo, minuto a minuto e hora a hora, resultado não encontrado para Bitcoin e Ethereum.

\subsection{Dash}

Como aponta o sumário estatístico presente na Tabela 7, a média do retorno logarítmico é próxima a zero e a volatilidade do retorno logarítmico do ativo é alta, para as agregações de tempo segundo (237,969 observações), minuto (111,781 observações), hora $(11,257$ observações) e dia (526 observações). A média de preço do ativo ficou em $\$ 106.85$ (agregação segundo), \$491.70 (agregação minuto), \$382.56 (agregação hora) e \$367.67 (agregação dia), com alta volatilidade de preços para todas as agregações de tempo.

Tabela 7 - Sumário estatístico do Dash

\begin{tabular}{c|c|c|c|c|c|c|c|c}
\hline & \multicolumn{2}{|c}{ Segundo } & \multicolumn{2}{c|}{ Minuto } & \multicolumn{2}{c|}{ Hora } & \multicolumn{2}{c}{ Dia } \\
\hline & Retorno & Preço & Retorno & Preço & Retorno & Preço & Retorno & Preço \\
\hline count & \multicolumn{2}{|c|}{237,969} & \multicolumn{2}{|c|}{111,781} & \multicolumn{2}{c|}{11,257} & \multicolumn{2}{|c}{526} \\
\hline mean & $\$ 0.000004$ & $\$ 106.85$ & $\$ 0.00001$ & $\$ 491.70$ & $\$ 0.00009$ & $\$ 382.56$ & $\$ 0.00206$ & $\$ 367.67$ \\
\hline std & $\$ 0.006333$ & $\$ 77.68$ & $\$ 0.00891$ & $\$ 318.06$ & $\$ 0.02477$ & $\$ 263.24$ & $\$ 0.06858$ & $\$ 259.48$ \\
\hline min & $-\$ 1.14862$ & $\$ 1.20$ & $-\$ 1.14862$ & $\$ 66.67$ & $-\$ 1.14862$ & $\$ 66.85$ & $-\$ 0.22925$ & $\$ 71.02$ \\
\hline & - & & & & & & & \\
$\mathbf{2 5 \%}$ & $\$ 0.000034$ & $\$ 46.00$ & $-\$ 0.00141$ & $\$ 230.00$ & $-\$ 0.00850$ & $\$ 193.00$ & $-\$ 0.03473$ & $\$ 189.21$ \\
\hline $\mathbf{5 0 \%}$ & $\$ 0$ & $\$ 74.70$ & $\$ 0.00000$ & $\$ 390.17$ & $\$ 0.00000$ & $\$ 302.56$ & $\$ 0.00036$ & $\$ 295.51$ \\
\hline $\mathbf{7 5 \%}$ & $\$ 0.000017$ & $\$ 163.00$ & $\$ 0.00126$ & $\$ 703.20$ & $\$ 0.00854$ & $\$ 478.81$ & $\$ 0.03558$ & $\$ 444.92$ \\
\hline $\mathbf{m a x}$ & $\$ 0.237091$ & $\$ 368.00$ & $\$ 0.22373$ & $\$ 1,570.00$ & $\$ 0.38105$ & $\$ 1,568.00$ & $\$ 0.34731$ & $\$ 1,524.00$ \\
\hline
\end{tabular}

Fonte: Elaboração própria.

Assim como ocorre com as outras criptomoedas, existe diferença nos resultados dos modelos dependendo da granularidade de tempo. Os resultados encontrados pelo modelo segundo a segundo (Tabela 8) apresentam as mesmas autocorrelações dos resultados segundo a segundo para o Bitcoin, Ethereum e Litecoin. O modelo segundo a segundo do Dash aponta que existe uma autocorrelação positiva significativa nas transações, evidenciado pelo lag do coeficiente das transações na estimativa da equação de transações e uma autocorrelação negativa significativa entre retornos, evidenciado pelo lag do coeficiente do retorno na estimativa da equação de retorno. O modelo apontou também a existência de autocorrelação negativa significativa entre lag do retorno e transações em $\mathrm{t}$, como aponta o coeficiente do lag do retorno na equação de transações. Como ocorre com Bitcoin e Ethereum, não foi verificada autocorrelação positiva para o modelo segundo a segundo entre o lag de transações na equação de retorno.

Tabela 8 - Resultado do modelo VAR proposto por Hasbrouck (1991) para o Dash

\begin{tabular}{l|c|c|c|c|c|c|c|c|c}
\hline & \multicolumn{2}{|c}{ Segundo } & \multicolumn{2}{c|}{ Minuto } & \multicolumn{2}{c}{ Hora } & \multicolumn{2}{c}{ Dia } \\
\hline & Coeficiente & Prob & Coeficiente & Prob & Coeficiente & Prob & Coeficiente & Prob \\
\hline Resultado da Equação de Transações (dummy) \\
\hline Trans. & $0.971135 * * *$ & 0.000 & $0.760284 * * *$ & 0.000 & $0.241705 * * *$ & 0.000 & $0.104152 * *$ & 0.020 \\
\hline & - & - & & - & & & \\
Retorno & $1.074801 * * *$ & 0.000 & $3.876753 * * *$ & 0.000 & $1.304055^{* * *}$ & 0.001 & 0.309763 & 0.631 \\
\hline Resultado da Equação de Retorno & - & 0.392 & $0.000085^{* * *}$ & 0.001 & $0.000491 * *$ & 0.039 & $0.006087 * *$ & 0.050 \\
\hline Trans. & 0.000011 & - & 0.000 & - & 0.000 & $-0.119970 *$ & 0.007 \\
\hline Retorno & - & 0.000 & - & & & \\
\hline
\end{tabular}


Efeito Feedback Trading em Criptomoedas com Dados de Alta Frequência

\begin{tabular}{l|l|l|l|l|l|l|l}
\hline & $0.136935 * * *$ & & $0.121065 * * *$ & $0.058621 * * *$ & & & \\
\hline
\end{tabular}

Fonte: Elaboração própria

O resultado encontrado para o modelo com granularidade minuto a minuto é similar aos resultados encontrados para o Bitcoin, Ethereum e Litecoin. O resultado do modelo minuto a minuto aponta que existe forte autocorrelação positiva entre volume de transações e o lag do volume de transações, forte autocorrelação positiva entre o lag do volume de transações e o retorno e forte autocorrelação negativa entre o lag do retorno e o retorno, em linha com o estudo de Hasbrouck (1991).

Assim como ocorre nos modelos do Bitcoin, Ethereum e Litecoin, além das correlações encontradas acima, o modelo evidencia a existência de forte autocorrelação negativa entre o lag do retorno e o fluxo de transação, indicando a existência de feedback trading negativo também para Dash.

O modelo hora a hora, aponta para as mesmas correlações do modelo minuto a minuto. O modelo dia a dia apresenta também resultados semelhantes ao modelo hora a hora, com nível de significância menor, com exceção da autocorrelação negativa entre retorno e transação, que não é significativa para os dados com granularidade dia a dia.

Como encontrado na análise dos dados para o Bitcoin, Ethereum e Litecoin, não foi encontrado o efeito de feedback trading positivo para o Dash em nenhuma granularidade de tempo. Observa-se pelos dados a existência de feedback trading negativo nas granularidades segundo a segundo, minuto a minuto e hora a hora, resultado encontrado apenas para o Litecoin e Dash.

Desta maneira, com o intuito de se realizar um fechamento das análises, optou-se por desenvolver um sumário de representação da direção da relação (positiva ou negativa) e as respectivas significâncias estatísticas (tabela 9).

Tabela 9 - Sumário da Análise de Feedback Trading das Criptomoedas

\begin{tabular}{|c|c|c|c|c|c|}
\hline & & Segundo & Minuto & Hora & Dia \\
\hline \multirow{4}{*}{ Bitcoin } & lag transação $\rightarrow$ equação transação & $* * *+$ & $* * *+$ & $* * *+$ & $* * *+$ \\
\hline & $\begin{array}{c}\text { lag retorno } \rightarrow \text { equação } \\
\text { transação }\end{array}$ & $* * *$ & $* * *$ & & \\
\hline & lag transação $\rightarrow$ equação retorno & & $* * *+$ & & \\
\hline & lag retorno $\rightarrow$ equação retorno & $* * *$ & $* * * \ldots$ & $* * *_{-}$ & \\
\hline \multirow{4}{*}{ Ethereum } & lag transação $\rightarrow$ equação transação & $* * *+$ & $* * *+$ & $* * *+$ & $* * *+$ \\
\hline & $\begin{array}{c}\text { lag retorno } \rightarrow \text { equação } \\
\text { transação }\end{array}$ & $* * *$ & $* * *$ _ & & \\
\hline & lag transação $\rightarrow$ equação retorno & & $* * *+$ & & \\
\hline & lag retorno $\rightarrow$ equação retorno & $* * *$ & $* * *$ & & \\
\hline \multirow{4}{*}{ Litecoin } & lag transação $\rightarrow$ equação transação & $* * *+$ & $* * *+$ & $* * *+$ & $* * *+$ \\
\hline & $\begin{array}{c}\text { lag retorno } \rightarrow \text { equação } \\
\text { transação }\end{array}$ & $* * *$ & $* * *$ & $* * *$ & \\
\hline & lag transação $\rightarrow$ equação retorno & & $* * *+$ & & \\
\hline & lag retorno $\rightarrow$ equação retorno & $* * *$ & $* * *$ & $* * *$ & $* * *$ \\
\hline Dash & lag transação $\rightarrow$ equação transação & $* * *+$ & $* * *+$ & $* * *+$ & $* *+$ \\
\hline
\end{tabular}


Efeito Feedback Trading em Criptomoedas com Dados de Alta Frequência

\begin{tabular}{l|c|c|c|c|c}
\hline & $\begin{array}{c}\text { lag retorno } \rightarrow \text { equação } \\
\text { transação }\end{array}$ & $* * * \ldots$ & $* * *_{-}$ & $* * *_{-}$ & \\
\cline { 2 - 6 } & lag transação $\rightarrow$ equação retorno & & $* * *+$ & $* *+$ & $* *_{+}$ \\
\cline { 2 - 5 } & lag retorno $\rightarrow$ equação retorno & $* * *-$ & $* * *_{-}$ & $* * * *_{-}$ & $*_{-}$ \\
\hline
\end{tabular}

Código para nível de significância: $1 \%$ ‘***' $5 \%$ ‘**’ $10 \%$ ‘*’

As linhas em negrito apontam o respectivo efeito do feedback trading. Ns espaços vazios, não houve significância estatística.

Fonte: Elaboração própria.

Ainda existem poucos estudos similares que utilizaram o efeito comportamental de feedback trading para se poder avaliar os resultados de forma comparativa. Apenas Silva (2020) analisou 50 criptoativos durante o período de 2013 - 2018 utilizando o modelo de Sentana e Wadhwani (1992) que incorpora as inovações trazidas por Bollerslev (1986) com o modelo GARCH. Ao se comparar os resultados para o BTC e ETH, foi possível notar que os resultados foram diferentes (apresentaram o efeito do feedback trading positivo).

Não é algo incomum, já que, assim como observado por Antoniou, Koutmos e Pericli (2005), diferentes modelos de negociação de feedback trading têm implicações diferentes para o padrão de autocorrelação dos retornos das ações. Por exemplo, os modelos de feedback trading usados por Shiller (1984), Cutler, Poterba e Summers (1990) e Sentana e Wadhwani (1992) implicam em uma autocorrelação positiva de retornos de curto prazo.

\section{CONCLUSÃO}

Este trabalho teve como objetivo entender como o efeito de feedback trading se manifesta nas criptomoedas Bitcoin, Ethereum, Litecoin e Dash usando o modelo VAR proposto por Hasbrouck (1991). Como visto na análise de resultados, é possível concluir que existem diferenças nos resultados dependendo se o dado é agregado por segundo, minuto, hora ou dia. Foi possível notar que existem diferenças nos resultados dependendo da criptomoeda avaliada.

Os resultados dos modelos segundo a segundo são similares para todas as criptomoedas e estão em linha com as descobertas feitas por Hasbrouck (1991). Como já evidenciado pelo autor, os dados apontam para as seguintes relações entre as equações de retorno e fluxo de transação. Para nenhuma criptomoeda no modelo segundo a segundo foi significativa a autocorrelação positiva entre o lag de transações no retorno em t. Esse ponto difere das descobertas feitas por Hasbrouck (1991).

Os resultados dos modelos minuto a minuto são similares para todas as criptomoedas e estão em linha com as descobertas feitas por Hasbrouck (1991). Como já evidenciado pelo autor, os dados apontam para as seguintes relações entre as equações de retorno e fluxo de transação:

- O lag de transações tende a exercer um efeito positivo no retorno em $t$, ou seja, os movimentos de preços seguem o fluxo de compra;

- As transações se mostraram positivamente autocorrelacionadas, sugerindo que compras tendem a levar a compras e vendas a vendas;

- Os retornos são negativamente autocorrelacionados;

- O efeito negativo do lag do retorno na estimativa da equação da transação implica na existência de causalidade Granger-Sims, partindo de revisões de cotação para transações. 
Efeito Feedback Trading em Criptomoedas com Dados de Alta Frequência

Os resultados para os modelos hora a hora e dia a dia apresentam resultados distintos dependendo do criptoativo analisado e, dependendo do criptoativo, não apresentam todas as correlações encontradas no estudo de Hasbrouck (1991).

O resultado encontrado na análise dos dados para o Bitcoin, Ethereum, Litecoin e Dash evidencia que não existe efeito de feedback trading positivo em nenhuma granularidade de tempo. Observa-se, por outro lado, a existência de feedback trading negativo na granularidade segundo a segundo e minuto a minuto para o Bitcoin, Ethereum, Litecoin e Dash e na granularidade hora a hora apenas para o Litecoin e Dash. Para a granularidade de tempo dia a dia não é observada a existência de feedback trading.

Uma hipótese sobre a existência de feedback trading negativo para as criptomoedas pode ser devido ao uso de bots (programas de computador que utilizam diversos indicadores para reconhecer tendências de mercado e executar negociações automaticamente) pelos investidores. Devido a alta volatilidade do mercado de criptomoedas, os bots ficaram muito populares entre investidores por permitir que eles tenham controle dos seus ativos o tempo todo, além de conseguirem executar negociações de maneira mais rápida e eficiente do que os investidores se estiverem com especificações corretas. Além disso, os bots não estão suscetíveis a negociações emocionais provocadas pela alta volatilidade do mercado de cripotoativos, o que pode explicar a presença de feedback trading negativo e não feedback trading positivo.

Uma análise sobre a existência de feedback trading comparando diferentes períodos de tempo pode ser um interessante tema de estudo para trabalhos futuros e pode evidenciar o amadurecimento da estratégia dos investidores para essas criptomoedas. Como evidenciado no estudo realizado por Pavel, Rajcaniova e D'Artis (2015) inicialmente, quando o Bitcoin era pouco conhecido entre os investidores, as buscas por informação sobre Bitcoin (visualizações na Wikipedia foram usados como proxie para esse fator) exerciam forte impacto no preço do Bitcoin. A longo prazo, entretanto, as visualizações na Wikipedia não impactam o preço do criptoativo. Isso pode indicar um amadurecimento dos investidores ao longo do tempo.

\section{REFERÊNCIAS}

ANTONIOU, A.; KOUTMOS, G.; PERICLI, A. Index futures and postive feedback trading: Evidence from major stock exchanges. Journal of Empirical Finance, v. 12, n. 2, p. 219238, 2005.

BOHL, M. T.; REITZ, S. The influence of positive feedback trading on return autocorrelation: Evidence for the German stock market. In GEBERL, S. (Eds.), Aktuelle Entwicklungen im Finanzdienstleistungsbereich, p. 221-233, 2004.

BOHL, M. T.; KLEIN, A. C.; SIKLOS, P. L. Short-selling bans and institutional investors' herding behaviour: Evidence from the global financial crisis. International Review of Financial Analysis, v. 33, n. 1, p. 262-269, 2014.

BOLLERSLEV, T. Generalized autoregressive conditional heteroskedasticity. Journal of Econometrics, v. 31, n. 3, p. 307-327, 1986.

BOUOIYOUR, J.; SELMI, R.; TIWARI, A. K.; OLAYENI, O. R. What drives Bitcoin price. Economics Bulletin, v. 36, n. 2, p. 843-850, 2016.

BUCHHOLZ, M.; DELANEY, J.; WARREN, J.; PARKER, J. Bits and bets, information, price volatility, and demand for Bitcoin. Economics, v. 1, n. 312, p. 1-48, 2012. 
CHANG, C. L.; KE, Y. P. Testing price pressure, information, feedback trading, and smoothing effects for energy exchange traded funds. Annals of Financial Economics, v. 9, n. 2, 1440006, 2014.

CHAU, F.; DEESOMSAK, R.; LAU, M. C. Investor sentiment and feedback trading: Evidence from the exchange-traded fund markets. International Review of Financial Analysis, v. 20, n. 5, p. 292-305, 2011.

CHAU, F.; KUO, J. M.; SHI, Y. Arbitrage opportunities and feedback trading in emissions and energy markets. Journal of International Financial Markets, Institutions and Money, v. 36, n. 1, p. 130-147, 2015.

COHEN, B. H.; SHIN, H. S. Positive feedback trading under stress: Evidence from the US Treasury securities market. [BIS working paper number 122]. Washington, DC: Meetings, 2013.

CUTLER, D. M.; POTERBA, J. M.; SUMMERS, L. H. Speculative dynamics and the role of feedback traders. The American Economic Review, v. 80, n. 2, p. 63- 68, 1990.

DANIELSSON, J.; LOVE, R. Feedback trading. International Journal of Finance \& Economics, v. 11, n. 1, p. 35-53, 2006.

GALlO, E. R. S. Economia Comportamental aplicada a Finanças e o Modelo de Agentes: um estudo sobre a presença da subjetividade humana na tomada de decisão e suas implicações no mercado acionário. Dissertação (Mestrado em Economia). Universidade Estadual Paulista (UNESP), São Paulo, 2016.

HASBROUCK, J. Measuring the information content of stock trades. The Journal of Finance, v. 46, n. 1, p. 179-207, 1991.

KAHNEMAN, D.; TVERSKY, A. Prospect Theory: An Analysis of Decision under Risk. Econometrica, v. 47, n. 2, p. 263-292, 1979.

. The Framing of Decisions and the Psychology of Choice. Science, v. 211, n. 4481, p. 453-458, 1981.

A., Rational Choice and the Framing of Decisions. The Journal of Business, v. 59, n. 4, p. 51-78, 1986.

LEASE, R. C.; LEWELLEN W. G.; SCHLARBAUM G. G. The Individual Investor: Attributes and Attitudes. The Journal of Finance, v. 29, n. 2, p. 413-433, 1974.

MUTH, J. F. Rational Expectations and the Theory of Price Movements. Econometrica, v. 29, n. 3, p. 315-335, 1961.

PAVEL, C.; MIROSLAVA, R.; D'ARTIS, K. The economics of BitCoin price formation. Applied Economics, v. 48, n. 19, p. 1799-1815, 2015.

SENTANA, E.; WADHWANI, S. Feedback traders and stock return autocorrelations: evidence from a century of daily data. The Economic Journal, v. 102, n. 411, p. 415-425, 1992.

SHILLER, R. J. Speculative prices and popular models. Journal of Economic Perspectives, v. 4, n. 2, p. 55-65, 1990.

SILVA, P.V.J.G. Ensaios sobre Moedas Digitais: Um Estudo sobre Volatilidade e Fenômenos Comportamentais. Tese (Doutorado em Administração de Empresas). Pontifícia Umiversidade Católica do Rio de Janeiro, Rio de Janeiro, 200 f., 2020. 
SILVA, P.V.J.G.; SANTOS, J. B.; PEREIRA, G. P. Behavioral Finance in Brazil: A Bibliometric Study from 2007 to 2017. Latin American Business Review, v. 20, n. 1, p. 6182, 2019.

SIMON, H. A. A Behavioral Model of Rational Choice. The Quarterly Journal of Economics, v. 69, n. 1, p. 99-118, 1955.

VAN WIJK, D. What can be expected from the BitCoin. [Working paper number 345986]. Rotterdam: Erasmus Universiteit, 2013.

WEINTRAUB, R. E., On Speculative Prices and Random Walks A Denial. Journal of Finance, v. 18, n. 1, p. 59-66, 1963.

\section{Apêndice 1}

- Endereço para acessar código em Python para coleta e armazenamento dos dados de criptomoedas da corretora Kraken:

https://colab.research.google.com/drive/1UHMPqUlk-fBdK0lT8ijjrwZB8GMsSsEJ 